\title{
INFLUÊNCIA DO TAMANHO DA PARCELA NA PRECISÃO DA FUNÇÃO DE DISTRIBUIÇÃO DIAMÉTRICA DE WEIBULL NA FLORESTA PRIMÁRIA DA AMAZÔNIA CENTRAL
}

\author{
Francisco Gasparetto Higuchi ${ }^{1}$, Joésio Deoclécio Pierin Siqueira ${ }^{2}$, Adriano José Nogueira Lima ${ }^{3}$, \\ Afonso Figueiredo Filho ${ }^{4}$, Niro Higuchi ${ }^{3}$ \\ ${ }^{1}$ Eng. Florestal, M.Sc., Doutorando em Engenharia Florestal, UFPR, Curitiba, PR, Brasil - fghiguchi@ gmail.com \\ ${ }^{2}$ Eng. Florestal, Dr., Depto. de Ciências Florestais, UFPR, Curitiba, PR, Brasil - joesio@ stcp.com.br \\ ${ }^{3}$ Eng. Florestal, Dr., INPA, Manaus, AM, Brasil - adriano@inpa.gov.br; niro@inpa.gov.br \\ ${ }^{4}$ Eng. Florestal, Dr., Depto. de Engenharia Florestal, UNICENTRO, Irati, PR, Brasil - afonso.figueiredo@pq.cnpq.br
}

Recebido para publicação: 01/11/2010 - Aceito para publicação: 24/04/2012

\begin{abstract}
Resumo
A função probabilística de Weibull é uma ferramenta importante para o manejo florestal. O objetivo deste trabalho foi avaliar se os dados da floresta amazônica se ajustam a essa função e se o tamanho da parcela influencia na sua consistência. Foram utilizados 15 tamanhos de parcelas diferentes, instaladas na Estação Experimental ZF - 2 do INPA em Manaus, com 10 repetições para os tamanhos menores e 5 para os maiores. Foram coletados os diâmetros das árvores adultas (DAP $\geq 10 \mathrm{~cm}$ ) e distribuídos em 11 classes diamétricas, com $10 \mathrm{~cm}$ de amplitude. Os parâmetros da função de Weibull foram estimados pelo método da máxima verossimilhança. Depois foi estimada a probabilidade da frequência esperada de cada classe diamétrica. A diferença entre a frequência esperada e a observada gerou um conjunto de qui-quadrados $\left(\chi^{2}\right)$ que foi comparado com o nível crítico de $5 \%(\alpha=0,05)$. Os resultados foram: (i) a função se ajustou ao conjunto de dados da floresta amazônica; (ii) o tamanho da parcela, estatisticamente, não apresentou influência na consistência do modelo; e (iii) pode-se usar uma única equação para florestas da região de Manaus.
\end{abstract}

Palavras-chave: Inventário florestal; estrutura diamétrica.

\begin{abstract}
Influence of plot size in Weibull's diameter distribution function precision in a primary forest in Central Amazon. This research aimed to evaluate how the observed data of Amazonian forest can be fitted to Weibull's probabilistic function and the influence of plot size to the fitness as well. The Weibull probabilistic function is an important tool for forest management. In this study it was used 15 different plot sizes, installed in the INPA's Experimental Site ZF2 in Manaus, replicated 5 times for the larger sizes and 10 times for the smaller ones. It was collected the diameter of adult trees (DBH > $10 \mathrm{~cm}$ ), and distributed into 11 diameter classes, with $10 \mathrm{~cm}$ interval. Weibull parameters were estimated by Maximum Likelihood method, and the probability of the expected frequency was estimated for each diameter class. The difference between observed and expected frequency generated a set of Chi-squared $\left(\chi^{2}\right)$ for a $5 \%$ critical level $(\alpha=0.05)$. The main results were: (i) the observed data were fitted by Weibull function appropriately; (ii) the precision obtained by the Weibull function was not influenced by plot size; and (iii) only one function is good enough for Manaus forests, independently of plot size.

Keywords: Forest inventory; diameter structure.
\end{abstract}

\section{INTRODUÇÃO}

Apesar de quedas nas taxas de desmatamento nos últimos anos, o mau uso do solo amazônico continua chamando a atenção da sociedade em geral. Até junho de 2009, o desmatamento acumulado era de $711.442 \mathrm{~km}^{2}$ ou $14 \%$ da Amazônia Legal (INSTITUTO NACIONAL DE PESQUISAS ESPACIAIS (INPE), 2010). Além do desmatamento, a exploração seletiva de madeira é também um importante responsável pela alteração da paisagem da Amazônia. 
Grande parte da exploração seletiva de madeira é feita sem autorização do órgão ambiental responsável, IBAMA e respectivos órgãos estaduais (HUMMEL, 2001). Essas explorações ilegais de madeira na Amazônia são, na maioria dos casos, realizadas sob uma baixa infraestrutura, em áreas de acesso limitado, e produzem madeira de baixa qualidade. Esses fatores fazem com que o Brasil deixe de participar de grande parte do mercado internacional, que, segundo Higuchi et al. (2006a), gira em torno de 50 milhões de $\mathrm{m}^{3}$ de madeira e que, em 1993, gerou US\$ 12 bilhões para os países exportadores de madeira.

Segundo Higuchi et al. (2006b), o cenário do mercado mundial tende a se voltar para a Amazônia. A Malásia Peninsular já dizimou totalmente as suas florestas primárias, e projeções indicam que a Ilha de Borneo (Sabah e Sarawak) devem ter o mesmo destino em 5 a 8 anos. A Indonésia, de acordo com Higuchi et al. (2006b), já deve ter esgotado suas reservas florestais. Por volta de 2015, restará apenas a Amazônia para suprir o mercado internacional de madeira dura tropical.

Com o mercado internacional focado na madeira da floresta amazônica, a produção de madeira pode se transformar em oportunidade de negócios para a região. Nessas condições, o valor da madeira em pé tende a aumentar. $\mathrm{O}$ manejo florestal poderá gerar muitos empregos diretos e poderá trazer investimentos para a região, principalmente para os municípios mais afastados dos centros metropolitanos. Porém, levando-se em conta o histórico de uso do solo amazônico, essa tendência do mercado pode ser considerada uma ameaça, porque, mesmo produzindo 25 milhões de $\mathrm{m}^{3}$ de madeira, a Amazônia Legal participou com apenas 7,2\% na composição do produto interno bruto (PIB) brasileiro (ano-base 2002), sendo que menos de $20 \%$ da madeira produzida vem de plano de manejo florestal. Além disso, segundo Higuchi (2006), há correlação positiva e significante entre produção de madeira e desmatamento e não existe correlação alguma entre produção de madeira e PIB per capita. O padrão atual de produção de madeira na Amazônia é, em síntese, predatório, porque incentiva o desmatamento e não distribui renda.

Para confirmar o cenário como oportunidade de negócios, são necessários métodos e ferramentas para atender às exigências legais e do mercado. Na Amazônia, a utilização do DAP como variável independente em equações de volume está bem consolidada. O próximo passo é disponibilizar uma função de distribuição de diâmetro para deixar o inventário florestal mais simples e mais barato. Este trabalho de pesquisa estudou a função de densidade probabilística de Weibull para estimar a distribuição de diâmetro.

Essa função foi introduzida no setor florestal por Bailey e Dell (1973). Existem dois tipos de função de Weibull: (i) com dois parâmetros (YANG et al., 1978; LAWRENCE; SHIER, 1981); e (ii) com três parâmetros (ZARNOCH et al., 1982; LITTLE, 1983; KRUG et al., 1984; ZARNOCH; DELL, 1985). Para ajustar a função de Weibull com 3 parâmetros existem dois métodos: (i) Percentil (PCT); e (ii) Máxima Verossimilhança (MLB), ambas apresentadas por Zarnoch e Dell (1985). Segundo os autores, a variância do método de MLB foi até quatro vezes superior à encontrada no PCT, porém gerando parâmetros melhores. Lawrence e Shier (1981) comentaram que a função de distribuição de Weibull é muito utilizada para aplicações práticas, mas não há registro algum na literatura que indique que o setor produtivo utilize esse tipo de função. A pergunta a responder é: essa técnica é consistente independentemente do tamanho da unidade de amostra utilizada?

Dessa forma, o objetivo do presente trabalho foi avaliar se a função de Weibull se ajusta aos valores observados, independentemente do tamanho da unidade amostral, e apresentar um modelo da função de Weibull que possa ser utilizado em qualquer conjunto de dados da Amazônia Central. A consistência do modelo foi verificada utilizando-se testes estatísticos paramétricos e não paramétricos.

\section{MATERIAL E MÉTODOS}

A área desse estudo localiza-se à margem esquerda da estrada vicinal ZF - 2, entre km-20 e km35, em terras da Estação Experimental de Silvicultura Tropical do INPA. A descrição da Estação, como um todo, foi detalhada por Jardim e Hosokawa (1986/87). As coordenadas geográficas do ponto inicial da parcela (estendendo à direita) são: $2^{\circ} 36^{\prime} 853^{\prime \prime}$ de latitude sul e $60^{\circ} 08^{\prime} 660^{\prime \prime}$ de longitude oeste (PINTO et al., 2003).

A parcela permanente possui 18 ha, tendo as seguintes dimensões: 300 metros de largura por 600 metros de comprimento. Está dividida em quinze linhas de 20 metros de largura por 600 metros de 
comprimento. Cada linha está dividida em 30 subparcelas de $20 \times 20 \mathrm{~m}$, com quatro quadrantes $(a, b, c$ e d). Esta parcela foi instalada pelo Projeto Jacaranda (INPA/Japan International Cooperation Agency JICA), para estudos de distribuição espacial e dinâmica da floresta primária (PINTO et al., 2003).

A vegetação da área de estudo é uma amostra representativa de uma floresta tropical úmida densa de terra firme amazônica. Área de "sub-região dos baixos platôs da Amazônia" foi a denominação dada por RADAMBRASIL (1978). As espécies dominantes entre árvores altas pertencem às famílias Fabaceae, Lecythidaceae e Sapotaceae.

O clima da região é quente e úmido (BASTOS et al., 1986), apresentando umidade relativa média muito alta, variando de 84 a $90 \%$. Segundo RADAMBRASIL (1978), as médias anuais de temperatura do ar variam de $25,6{ }^{\circ} \mathrm{C}$ a $27,6^{\circ} \mathrm{C}$. O tipo climático é $A m$ na classificação de Köppen.

Os solos encontrados nas bacias hidrográficas da ZF - 2 são do tipo Latossolos Amarelos Álicos, argilosos, que ocupam as superfícies dos platôs, sendo o estádio de referência sob floresta caracterizado pela presença de um horizonte médio, poroso, fortemente microagregado, situado entre dois horizontes menos porosos. Além disso, o material original desses solos é composto pelos sedimentos terciários do Grupo Barreiras, que são, nesse caso, essencialmente constituídos de minerais resistentes à alteração, tais como a caolinita, o quartzo, os óxidos e os hidróxidos de ferro e alumínio (CHAUVEL, 1982).

Foram coletados os diâmetros (DAP) de todas as espécies arbóreas dentro da parcela permanente com DAP $\geq 10 \mathrm{~cm}$. Do arquivo original de Pinto et al. (2003), foram excluídos da amostragem as palmeiras e os cipós. Foram simulados 13 tamanhos de parcelas diferentes (Tabela 1).

Tabela 1. Tamanhos das parcelas, área e número de repetições.

Table 1. Samples sizes, area and number of repetition.

\begin{tabular}{lcc}
\hline Tamanho da UA $(\mathbf{m} \times \mathbf{~ m})$ & Área $(\mathbf{h a})$ & $\mathbf{N}^{\mathbf{0}}$. de repetições \\
\hline $20 \times 50$ & 0,1 & 10 \\
$20 \times 100$ & 0,2 & 10 \\
$20 \times 200$ & 0,4 & 10 \\
$20 \times 300$ & 0,6 & 10 \\
$20 \times 600$ & 1,2 & 10 \\
$30 \times 100$ & 0,3 & 10 \\
$30 \times 300$ & 0,9 & 10 \\
$30 \times 600$ & 1,8 & 10 \\
$50 \times 100$ & 0,5 & 10 \\
$50 \times 200$ & 1,0 & 10 \\
$50 \times 300$ & 1,5 & 10 \\
$100 \times 100$ & 1,0 & 5 \\
$200 \times 200$ & 4,0 & 5 \\
\hline
\end{tabular}

UA: unidade amostral.

A principal questão deste trabalho foi saber se a função de distribuição de Weibull 3P mantém uma precisão aceitável no ajuste da distribuição de diâmetros da floresta primária e, ao mesmo tempo, saber se essa função mantém a consistência com tamanhos diferentes de unidades amostrais. Para cada parcela de cada tamanho (tratamento), há uma função ajustada. Depois disso, foram realizadas as comparações por meio da análise de variância (ANOVA), utilizando-se os valores de qui-quadrado $\left(\chi^{2}\right)$.

A função de Weibull 3P foi ajustada por meio do método da máxima verossimilhança usando-se um pacote estatístico no programa Microsoft Excel®. A probabilidade da frequência esperada pôde ser determinada por meio da função integrada de Weibull, apresentada por Zarnoch et al. (1982), como apresentada a seguir:

Em que: $a=$ parâmetro de localização;

$$
F(x)=1-\exp \left\{-\left[\frac{(x-a)}{b}\right]^{c}\right\}
$$

$b=$ parâmetro de escala;

$c=$ parâmetro de forma. 
Para a validação do modelo, foi aplicado o teste $\chi$, calculado para verificar se a distribuição estimada pela função de distribuição de Weibull segue a mesma distribuição observada. Depois foi comparado com o valor crítico $c$ com $(\mathrm{k}-1)$ graus de liberdade, sendo $\mathrm{k}=$ número de classes de diâmetro para um nível crítico de $5 \%(\alpha=0,05)$. Para calcular o $\chi^{2}$, utilizou-se a seguinte equação:

$$
\chi^{2}=\sum\left[\frac{(O i-E i)^{2}}{E i}\right]
$$

Em que: $\chi^{2}=$ qui-quadrado;

$\mathrm{O}_{\mathrm{i}}=$ frequência observada através do levantamento de campo;

$\mathrm{E}_{\mathrm{i}}=$ frequência esperada estimada pela função.

As hipóteses testadas foram:

$\mathrm{H}_{0}$ : Os dados observados seguem a distribuição Weibull.

$\mathrm{H}_{1}$ : Os dados observados não seguem a distribuição Weibull.

Caso o valor calculado de $\chi^{2}$ seja maior do que o valor tabelado, em um nível crítico de $5 \%$ $(\alpha=0,05), \mathrm{H}_{0}$ é rejeitada. Para confrontar essa hipótese, os DAPs das árvores (DAP $\geq 10 \mathrm{~cm}$ ) foram ordenados de maneira crescente e agrupados em 11 classes diamétricas, com amplitude de $10 \mathrm{~cm}$, trancando em árvores com diâmetro igual ou superior a $110 \mathrm{~cm}$. Dessa forma, o número de indivíduos na última classe é $\geq 5$, com base nos resultados de Barros (1980).

O passo seguinte foi verificar se essa função é consistente, independentemente do tamanho da unidade amostral. As seguintes hipóteses foram testadas:

$\mathrm{H}_{0}: \mu_{1}=\mu_{2}=\mu_{3}=\ldots=\mu_{15}$

$\mathrm{H}_{1}$ : pelo menos uma média é diferente.

Para se avaliar os testes de hipóteses, foi aplicado o teste $\mathrm{F}$ da análise de variância. Nesse caso, a variável dependente foi o valor calculado de $\chi^{2}$. As médias são os valores de $\chi^{2}$ calculados para cada tratamento.

Foi ajustada uma função de distribuição diamétrica para cada tratamento, utilizando-se os respectivos parâmetros médios estimados. Foi gerada uma equação genérica para cada tamanho de parcela e aplicada em uma parcela sorteada de cada tamanho, o que gerou 13 valores de $\chi^{2}$.

No caso de probabilidades baixas (em torno de $5 \%$ ), $\mathrm{H}_{0}$ é rejeitada, sendo aplicado o teste post hoc de Tukey, para identificar quais são os tamanhos diferentes. Nesse caso, foi utilizado o modelo alternativo, apresentado por Neter e Wasserman (1974), que tem o seguinte modelo linear aditivo:

$$
Y_{i j}=\mu .+\tau_{j}+\varepsilon_{i j}
$$

Em que: $\mu .=$ a constante comum a todas as observações;

$\tau_{j}=$ o efeito do j-ésimo nível do fator;

$\varepsilon_{i j}=$ independente $N\left(0, \sigma^{2}\right)$;

$i=1, \ldots, n_{j} ; j=1, \ldots, \mathrm{r}$.

Para a análise de variância, foram considerados 13 tamanhos, totalizando 120 repetições. Os graus de liberdade foram 12 entre tratamentos, 107 dentro dos tratamentos e 119 para o total. O teste-F foi calculado da seguinte maneira: $\mathrm{F}=\mathrm{MQE} / \mathrm{MQD}$. A probabilidade exata do teste foi calculada pelo software SYSTAT 8.0.

\section{RESULTADOS E DISCUSSÃO}

A distribuição diamétrica da população encontrada na área de estudo apresentou o comportamento esperado de toda floresta tropical natural, com muitos indivíduos nas primeiras classes 
(DAP $\leq 30 \mathrm{~cm}$ ) e poucos nas últimas (DAP $\geq 50 \mathrm{~cm}$ ). Quase $93 \%$ dos indivíduos levantados no inventário encontram-se nas três primeiras classes de diâmetro, caracterizando a distribuição em "J" invertido.

Os parâmetros da função de Weibull foram ajustados para cada tamanho de parcela. Depois foi realizada a validação do modelo. Os valores médios dos parâmetros de cada tratamento e seus respectivos valores médios de $\chi^{2}$ seguem listados na tabela 2 .

Tabela 2. Valores médios dos parâmetros da função de Weibull 3P ajustada para cada tamanho de parcela.

Table 2. Average values of the parameters of the Weibull 3P adjusted for each sampling unit.

\begin{tabular}{lcccc}
\hline \multirow{2}{*}{ Tratamento } & \multicolumn{3}{c}{ Parâmetros } & \multirow{2}{*}{$\chi^{\mathbf{2}}$} \\
\cline { 2 - 4 } $20 \times 50$ & 10,0 & 11,09947 & 0,89045 & 3,54263 \\
$20 \times 100$ & 10,0 & 10,57021 & 0,87633 & 5,19866 \\
$20 \times 200$ & 10,0 & 9,82307 & 0,80204 & 5,73630 \\
$20 \times 300$ & 10,0 & 9,85943 & 0,81421 & 6,93234 \\
$20 \times 600$ & 10,0 & 10,23524 & 0,85065 & 6,15582 \\
$30 \times 100$ & 10,0 & 10,16199 & 0,82199 & 6,03875 \\
$30 \times 300$ & 10,0 & 10,26098 & 0,84442 & 8,53217 \\
$30 \times 600$ & 10,0 & 9,98083 & 0,84124 & 8,04258 \\
$50 \times 100$ & 10,0 & 10,06107 & 0,81019 & 6,94767 \\
$50 \times 300$ & 10,0 & 9,93564 & 0,82912 & 7,25891 \\
$50 \times 600$ & 10,0 & 9,93494 & 0,84085 & 6,19292 \\
$100 \times 100$ & 10,0 & 9,93086 & 0,82175 & 5,60707 \\
$200 \times 200$ & 10,0 & 9,84729 & 0,83371 & 7,70499 \\
\hline Média geral & 10,0 & 10,13085 & 0,83669 & 6,45314 \\
\hline
\end{tabular}

A figura 1 ilustra a frequência observada em relação à esperada, calculada por meio da equação usando a média dos parâmetros ajustados para os diferentes tamanhos de parcela. Os valores estimados são muito próximos aos valores reais que, empiricamente, demonstra que a função se ajustou muito bem aos dados levantados.

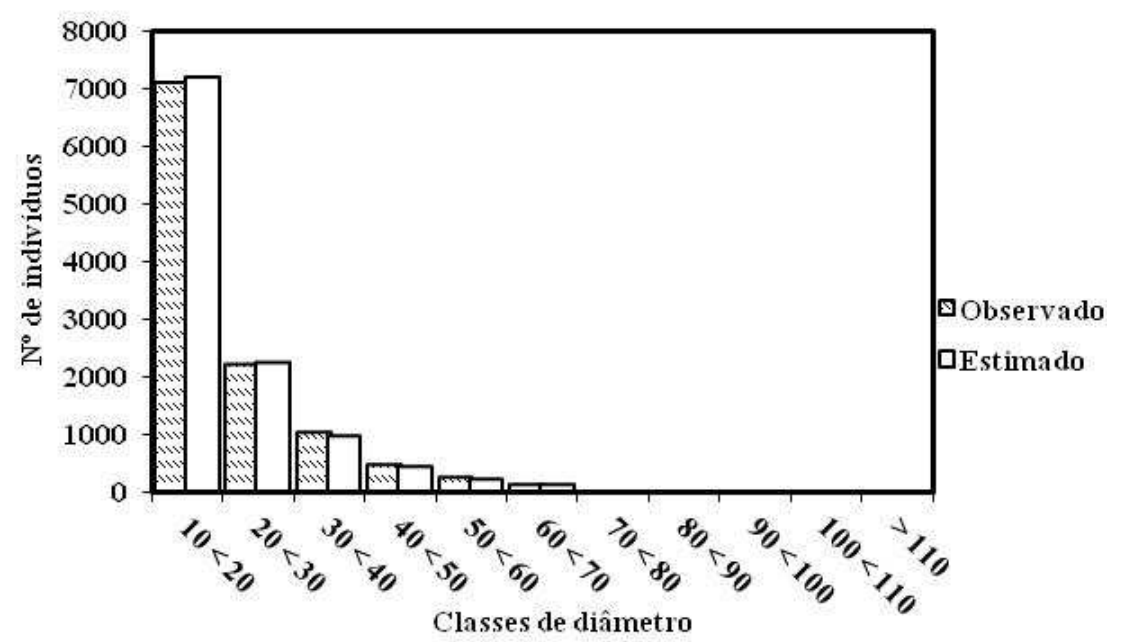

Figura 1. Frequência estimada e observada.

Figure 1. Estimated and observed frequency.

Esses resultados comprovam que a função de distribuição Weibull ajusta bem os valores observados de diâmetro em florestas primárias da Amazônia, independentemente do tamanho e forma da 
unidade amostral. Da mesma maneira, confirmam também os resultados obtidos anteriormente em áreas próximas da área de estudo (HIGUCHI, 1987; UMAÑA; ALENCAR, 1998).

Para avaliar a consistência da função, uma equação foi ajustada para cada tratamento, por meio de seus respectivos parâmetros médios, e foi aplicada em uma parcela sorteada de cada tamanho diferente. A figura 2 ilustra os valores de $\chi^{2}$ produzidos pelo processo de validação. Todos os $\chi^{2}$ gerados ficaram abaixo do nível crítico.

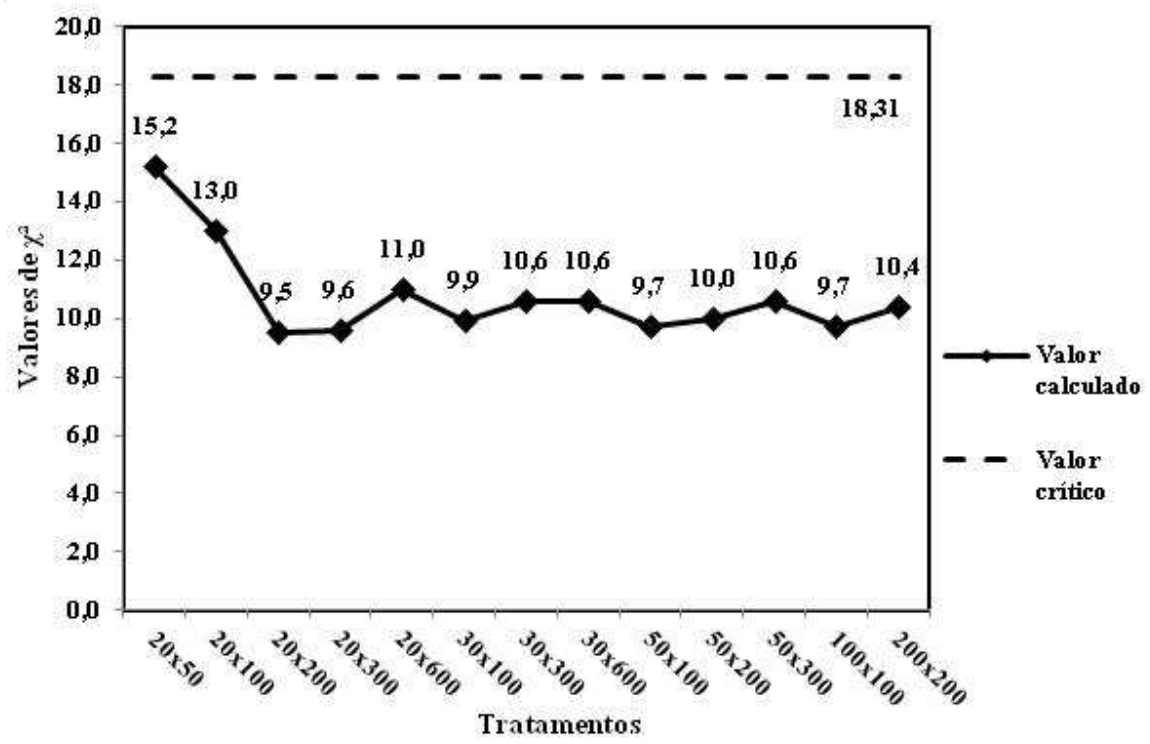

Figura 2. Valores dos $\chi^{2}$ gerados no processo de validação da função de Weibull.

Figure 2. Values of the $\chi^{2}$ generated in the process of validation of the Weibull's function.

A análise de variância (ANOVA) resultou em uma probabilidade do valor de $\mathrm{P}$ calculado sob a curva-P de 0,472. Evidência muito fraca para afirmar que há diferença estatística entre os tratamentos. Logo, não houve necessidade de realizar o Teste post hoc de Tukey. A variável dependente do modelo foi o $\chi^{2}$ calculado em cada tratamento.

A ANOVA confirmou que o desempenho da função não depende do tamanho da parcela. Como as evidências são fracas (teste-F; $\mathrm{p}=0,472$ ) para afirmar que os tamanhos diferentes têm desempenhos diferentes, fica a recomendação de utilizar os tamanhos de parcelas definidos na literatura (QUEIROZ, 1977; HIGUCHI et al., 1982). Atualmente, os inventários florestais realizados na Amazônia utilizam parcelas retangulares de $2.500 \mathrm{~m}^{2}$, dimensionadas em 20 metros de largura e 125 metros de comprimento.

\section{CONCLUSÃO}

O presente estudo conclui que: (i) como previsto na literatura, o método da máxima verossimilhança (ZARNOCH et al., 1982), de estimação dos parâmetros da função de Weibull 3P, é eficaz; (ii) os dados observados seguem a distribuição probabilística de Weibull; (iii) a função de Weibull 3P mostrou-se consistente, independentemente do tamanho da parcela, para a estimação da probabilidade da frequência por classe diamétrica; e (iv) como os diferentes tamanhos de parcela foram não significantes, é razoável utilizar as médias de a $(10,0)$, b $(9,665)$ e c $(0,847)$ para montar uma função genérica para a região de Manaus:

$$
F(x)=1-\exp \left\{-\left[\frac{(x-10)}{9,665}\right]^{0,847}\right\}
$$

para $x \geq a \geq 0, b>0$ e $c>0$ 


\section{REFERÊNCIAS}

BAILEY, R. L.; DELL, T. R. Quantifying diameter distributions with the Weibull function. Forest Science. Lawrence, v. 19, p. 97 - 104, 1973.

BARROS, P. L. C. Estudo das distribuições diamétricas da floresta do planalto Tapajós - Pará. 123f. Dissertação (Mestrado em Manejo Florestal) - Universidade Federal do Paraná, Curitiba. 1980.

BASTOS, T. X.; ROCHA, E. J. P.; ROLIM, P. A. M.; DINIZ, T. D. A. S.; SANTOS, E. C. R.; NOBRE, R. A. A.; CUTRIM, E. M. C.; MENDONÇA, R. L. D. The climate of the Brazilian Amazon Region for Agricultural Purposes; a State-of-the-art. In: $1^{\circ}$ Symposium On The Humid Tropics, 1, 1984, Belém, Pará. Anais... EMBRAPA-CPATU. 1986. p. 19 - 36.

CHAUVEL, A. Os latossolos amarelos, álicos, argilosos, dentro dos ecossistemas das bacias experimentais do INPA e da região vizinha. Acta Amazonica, Manaus, v. 12, n. 3, p. 47 - 60, 1982.

HIGUCHI, N. Short - term growth of an undisturbed tropical moist forest in the Brazilian Amazon. 129f. Tese (Doutorado em Manejo Florestal) - Michigan State University. East Lansing. 1987.

HIGUCHI, N., SANTOS, J. dos; JARDIM, F. C. S. Tamanho de parcela amostral para inventários florestais. Acta Amazonica, Manaus, 12(1): p. 91 - 103, 1982.

HIGUCHI, N.; SANTOS, J. dos; LIMA, A. J. N.; TEIXEIRA, L. M.; CARNEIRO, V. M. C.; TRIBUZY, E. S. Manejo florestal sustentável na Amazônia brasileira. Manaus, Apostila, p. 140 - 155, 2006 a.

HIGUCHI, N.; SANTOS, J. dos; MARTINS, L. T.; NOGUEIRA, A. J. L. O mercado Internacional de madeira tropical está à beira do colapso. SBPN Scientific Journal, São Paulo, 1 - 2: p. 33 - 41, 2006 b.

HUMMEL, A. C. Normas de acesso ao recurso florestal na Amazônia Brasileira: no caso do manejo florestal madeireiro. 104f. Dissertação (Mestrado em Ciências Florestais) - Programa de Biologia Tropical e Recursos Naturais do Convênio INPA/Univ. do AM. Manaus. 2001.

INSTITUTO NACIONAL DE PESQUISAS ESPACIAIS (INPE). <www.inpe.br>. Acesso em 2010.

JARDIM, F. C. S.; HOSOKAWA, R. T. Estrutura da floresta equatorial úmida da estação experimental de silvicultura tropical do INPA. Acta Amazonica, Manaus, $16 / 17$ (único): p. 411 - 507, 1986/87.

KRUG, A. G.; NORDHEIM, E. V.; GIESE, R. L. Determining initial values for parameters of a Weibull model: a case study. Forest Science. Lawrence, v. 30, n. 3, p. 573 - 581, 1984.

LAWRENCE, K. C.; SHIER, D. R. A comparison of least square and least absolute deviation regression models for estimating Weibull parameters. Communications in Statistics - Simulation and Computation, v. 10 , i. 3 , p. $315-326,1981$.

LITTLE, S. N. Weibull diameter distribution of mixed stands of western conifers. Canadian Journal of Forest Research, Otawa, v. 13, p. 85 - 88, 1983.

NETER, J.; WASSERMAN, W. Applied linear statistical model: Regression, Analysis of Variance and Experimental Designs. Homewood, Illinois, USA. Richard D. Irwin, INC., 1974, 842 p.

PINTO, A. C. M.; HIGUCHI, N.; IIDA, S.; SANTOS, J.; RIBEIRO, R. J.; ROCHA, R. M.; SILVA, R. P. Padrão de distribuição espacial de espécies florestais que ocorrem na região de Manaus - AM. Projeto Jacaranda Fase II - Pesquisas Florestais na Amazônia Central (editado por HIGUCHI et al.). Manaus, p. 1 - 20, 2003.

QUEIROZ, W. T. Efeitos da variação estrutural em unidade amostral na aplicação do processo de amostragem em conglomerados nas florestas do Planalto do Tapajós. Floresta, Curitiba, 8(1): p. 19 - 23, 1977.

RADAMBRASIL. 1978. Programa de Integração Nacional. Levantamentos de Recursos Naturais. v. 18 (Manaus) - RADAM (projeto) DNPM, Ministério das Minas e Energia. Brasil. 626 pp. 
UMAÑA, C. L. A.; ALENCAR, J. C. Distribuições diamétricas da floresta tropical úmida em uma área no município de Itacoatiara - AM. Acta Amazonica. Manaus, 28(2): p. 167 - 190, 1998.

YANG, R. C.; KOZAK, A.; SMITH, J. H. G. The potential of Weibull-type functions as flexible growth curves. Can. J. For. Res. Vancouver - BC. v. 8, p. 424 - 431, 1978.

ZARNOCH, S. J.; RAMM, C. W.; RUDOLPH, V. J.; DAY, M. W. The effects of Red Pine thinning regimes on diameter distribution fitted to the Weibull function. Research Report, Michigan State Univ. Jan. 1982.

ZARNOCH, S. J.; DELL, T. R. An evaluation of Percentile and Maximum Likelihood estimators of Weibull parameters. Forest Science. Lawrence, v. 31, n. 1, p. 260 - 268, 1985. 